\title{
Replacing Chemical Nematicides Using Biological Tools
}

\section{Opinion}

A major global challenge in the coming years will be to ensure food security and to feed the increasing human population. Now, we need to increase the sustainability of agricultural productivity to meet the increasing demand for food. According to Indian Agriculture Report 2012-2013, India has about 2.4\% and 4\% of the world's geographical area and water resources respectively, but has to support about $17 \%$ of the world's human population and $15 \%$ of the livestock. Agriculture is an important sector of the Indian economy, accounting for $14 \%$ of the nation's gross domestic product (GDP), about $11 \%$ of its exports. Root-knot disease caused by root-knot nematode is an important factor which declines our crop yields, particularly in tomato it reduces more than $27 \%$ yield annually.

Nematicides of chemical origin are widely used for the management of root-knot nematodes; large scale use of such chemical nematicides poses environmental hazards, besides being costly and uneconomical. Many bioinoculants are being used for controlling large numbers of soil borne diseases including the root-knot disease. Large numbers of microbes have been evaluated for controlling such diseases among these microbes Arbuscular mycorrhizal (AM) fungi and plant growth promoting rhizobacteria (PGPRs) have the greater potential for controlling it and hence they are one of the important biocontrol agents which could be used in the place of chemical nematides in agricultural fields. These organisms are economically and environmentally secure biocontrol tools against the soil borne pathogens. Generally all these organisms including root-knot nematodes live in the rhizosphere of the plants where they share the same niche. Rootknot nematodes affect all economically important characters of plants viz. yield, growth, hight, etc. This pathogen generally causes stress to the plants because of its damaging effect on root cells. AM and PGPRs affect the life cycle of nematodes because they compete with the nematode inside the root cells and provide some resistance to the plants.

Opinion
Volume 9 Issue 3 - 2017
Ishwar Prakash Sharma*
Patanjali Herbal Research Department, Patanjali Research
Institute, India
*Corresponding author: Ishwar Prakash Sharma,
Patanjali Herbal Research Department, Patanjali Research
Institute, Haridwar-249 405, Uttarakhand, India, Tel: +91
7579095587; Email: ipsharma.com@gmail.com
Received: October 30, 2017 | Published: November 14,
2017

To check the effect of both bioinoculants on the nematode parasitism, an experiment was conducted in which tomato plants selected as experimental plants [1]. In the beginning, tomato seeds were surface disinfected by immersion into $70 \%$ ethanol and $3 \%$ sodium hypochlorite followed by washing with sterile water. After sterilization, the seeds were sown in the autoclaved sand. For this two nurseries (one with mycorrhiza and other without mycorrhizal culture) were prepared. Mycorrhizal culture was inoculated during seeds sowing in nursery; a thin layer of mycorrhizal soil from maize rhizosphere was inoculated in the pure sterilized sand followed by sowing of sterilized tomato seeds in the inoculums. For non-mycorrhizal plants, the same nursery was prepared without mycorrhiza. Hoagland solution [2] was given weekly in both the nurseries. After four leaves stage the plants were transplanted into the 1:1 ratio sand and soil inoculated with the plant growth promoting rhizobacteria, fluorescent pseudomonad strains R62 and R81. Ten days after transplanting 500 freshly hatched nematode second stage juveniles (J2) were inoculated near the roots, the observations were taken after 30 days of the $\mathrm{J} 2$ inoculation.

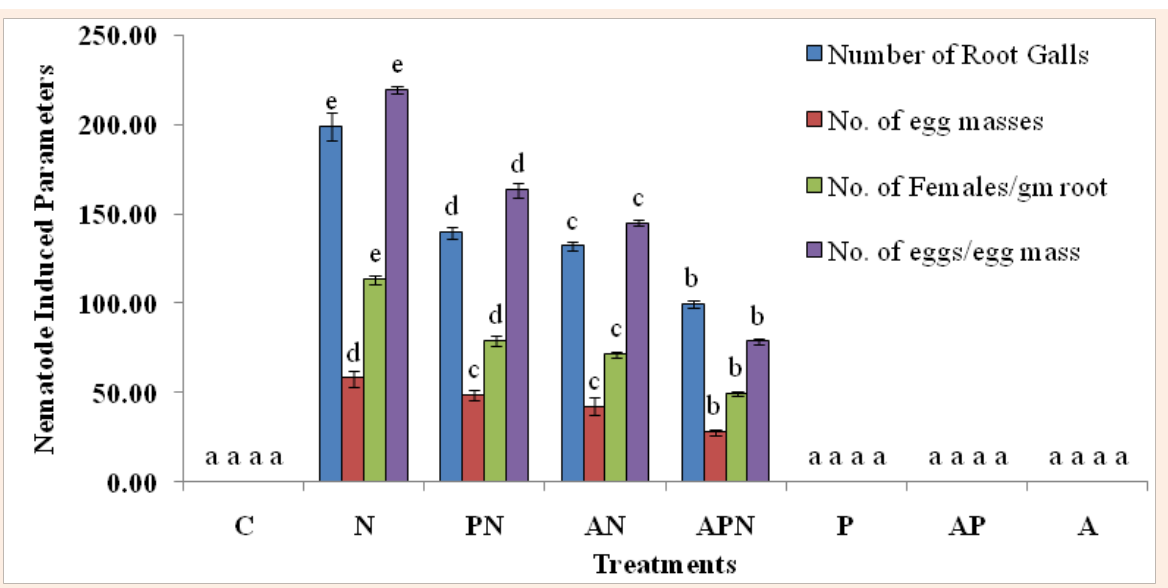

Figure 1: Effect of selected bioinoculents on nematode induced parameters.

C: Control; N: Nematodes; P: PGPRs; A: Arbuscular Mycorrhiza 'AM'. Error bars represent standard error of three replicates. Different letters indicate a significant difference $(\mathrm{P}<0.05)$ of treatment. 
Nematode induced parameters, numbers of root galls (49.92\%, $33.67 \%$ and $29.82 \%)$, egg masses $(51.72 \%, 27.01 \%$ and $16.09 \%)$, adult females $(56.47 \%, 37.06 \%$ and $30 \%)$ and eggs per egg mass $(64.13 \%, 33.89 \%$ and $25.53 \%)$ reduction were found in coinoculation (AM and PGPR) followed by in AM and PGPR treated plants, respectively over nematode infected plants. All data have been represented in Figure 1. In nutshell, it may be concluded in this experiment that both of the biocontrol agents i.e. AM and PGPRs are useful for the management of the root-knot disease in the agricultural crops and also they act as an economically and environmentally secure biocontrol tool to replace the chemical nematicides. Hence, application of these biocontrol agents should be encouraged for better agricultural productivity and for sustaining a secured environment as well.

\section{References}

1. Sharma IP, Sharma AK (2017) Physiological and biochemical changes in tomato cultivar PT-3 with dual inoculation of mycorrhiza and PGPR. Symbiosis 71(3): 175-183.

2. Hoagland DR, Arnon DI (1950) The water-culture method for growing plants without soil. Calif Agr Expt Sta 347: 1-32. 